\title{
Laser-induced Plasma Investigations during Material Processing
}

\author{
David DIEGO-VALLEJO ${ }^{* 1,2}$, Manuela SCHWAGMEIER ${ }^{* 3}$, David ASHKENASI ${ }^{* 3}$, Gerd ILLING $^{* 3}$, Hans Joachim EICH- \\ $\operatorname{LER}^{* 1,3}$ \\ ${ }^{* 1}$ Technical University of Berlin, Optics Institute, Straße des 17. Juni 135, 10623 Berlin, Germany \\ *2 National Polytechnic Institute, Av. Instituto Politécnico Nacional S/N, 07738 Mexico City, Mexico \\ ${ }^{* 3}$ Laser- und Medizin- Technologie Berlin GmbH (LMTB), Applied Laser Technology, Fabeckstr. \\ 60-62, 14195 Berlin, Germany \\ E-mail:d.ashkenasi@lmtb.de
}

\begin{abstract}
Laser-induced breakdown spectroscopy (LIBS), a versatile analytical tool to determine sample compositions, has recently been applied to monitor and control laser material processing. By observing the induced plasma during laser machining, an insight of the current processing stage can be gained. The aim of this project is to use the already present plasma in laser material processing to improve the quality of the product by means of spectroscopic analysis. Experimental studies have been carried out looking for useful correlations between material processing parameters and spectroscopic plasma signal. The presence of such characteristic patterns in the spectral data can be utilized to implement a control strategy to monitor the process.
\end{abstract}

DOI:10.2961/jlmn.2011.02.0009

Keywords: Laser processing, mechanical laser trepanning, LIBS, linear correlation.

\section{Introduction}

Advanced laser material processing is a high precision and accuracy machining procedure that presents several advantages over mechanical and chemical methods due to its non-contact, fast and versatile performance, including the use of sensors to control its performance, improving the quality of the end product.

When an energetic laser beam is focused onto a surface, several phenomena take place, including material removal and plasma induction. The latter can be analyzed by means of LIBS, that stands for Laser-Induced Breakdown Spectroscopy. This technique is based on the dissociation of the particles of the ablated material and the ionization of the surrounding environment by the effect of laser radiation, creating a plasma that is optically collected to produce a spectrum of emission lines of atomic, ionized and molecular constituents of the sample. Hence, LIBS can provide analytical information about its elemental composition and different physical and chemical features [1-3].

Some advantages of LIBS over other analytical techniques are: real-time and in-situ measurement due to the non-existent or little sample preparation, multiple element detection capability regardless the aggregation state of the sample and the number of constituents, among others. Based on this technique, several applications have been developed like depth profiling, chemical imaging, soil characterization, authentication of artworks, and recently, monitoring and control of laser material processing [1-15].

By observing the induced plasma during laser machining an estimation of the current processing condition can be obtained as a control mechanism for several applications including thin layer selective removal, artwork preservation, on-line identification, quality control and some other [4-15].

Spectroscopic techniques used to estimate the amount of ablated material are based on analyzing emission line intensity and on characteristic fingerprint discrimination. The former is mainly based on the individual analysis of different emission lines present in the spectrum. Frequency shifting and broadening of these lines are evaluated and compared to obtain the relevant information [2,3,12-20].

As every material presents a characteristic emission spectrum under similar excitation conditions, its use as a fingerprint to identify the material being affected based on linear or rank correlation algorithms has increased [4-8].

Spectra are read and then compared with a database of pre-recorded data to obtain information about how similar the sample and the reference are, and then, a discrimination process is applied. By utilizing these recognition algorithms, different groups have shown the feasibility of enhancing the material removal based on LIBS analysis, creating close-looped systems based on the degree of resemblance of a sample compared with a reference $[4,7,8,10,12$ 15].

As one example, thin-layer removal monitoring by means of LIBS analysis is possible when a change in spectroscopic signal read while machining different layers of the sample, then the ablation process is stopped or decreased [4,8,11,13,14,17].

Moreover, laser processing can be enhanced by means of mechanical trepanning, a technique in which the laser spot is moved in a circular path to produce a hole with a diameter bigger than the beam waist of the focused beam. This effect is produced by using a rotating optical setup in order to improve the quality of the machining in terms of speed and quality.

Laser-induced plasma analysis and the use of a trepanning system during material processing has not been reported to date, that is why the current work is devoted to the analysis and possible application of on-line controlling strategies to a laser machining system that includes trepanning optics. The study is concerned in its first stage with processing of ceramic materials.

\section{Experimental}


The experimental setup used in this work is represented in Fig. 1.

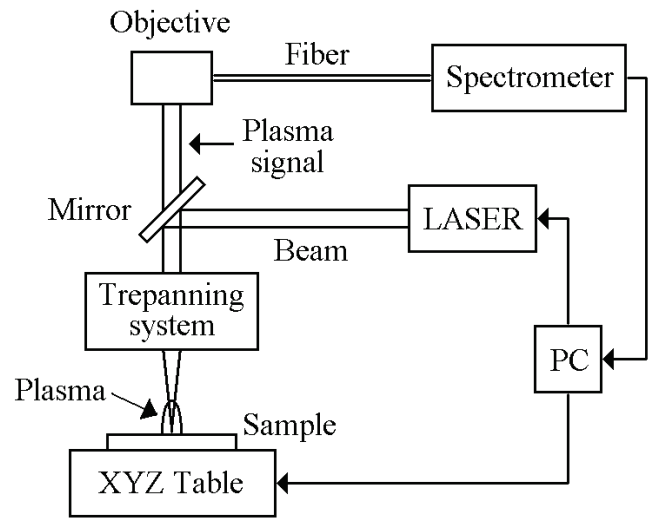

Fig. 1 Schematic of the experimental setup for LIBS analysis during material processing.

The laser used to produce both, the plasma and the processing was a LMTB lamp-pumped Q-switch Nd:YAG laser operated at its fundamental wavelength of $1064 \mathrm{~nm}$ working with pulse duration of $150 \mathrm{~ns}$ at variable pulse repetition rate and output energy. For the current experiment, an energy of $1 \mathrm{~mJ}$ per pulse and a $5 \mathrm{kHz}$ pulse frequency were used unless otherwise specified.

Laser radiation was guided to the working points by a set of dielectric mirrors with infra-red high-reflectance coating. Before reaching the sample surface, the beam was guided through a novel LMTB laser trepanning system that focused the incoming beam using a $75 \mathrm{~mm}$ lens and created a circular pattern, distributing the delivered energy onto the sample surface. A more detailed description of the used trepanning setup can be found in [21]. A moderate rotation speed of $500 \mathrm{rpm}$ was chosen.

The samples were placed on an Aerotech CNC motion stage with a $10 \mu \mathrm{m}$ resolution to produce the desired processing patterns by means of interpolated displacement of $\mathrm{XY}$ axes. Z-axis determined the perpendicular distance from sample to focusing optics.

The laser-induced plasma was gathered using a $0.75 \mathrm{x}$ microscope objective under on-axis configuration to focus the signal into a $400 \mu \mathrm{m}$ optical fiber connected to a miniature spectrometer. The spectrometer was an Ocean Optics USB2000+UV-VIS presenting a resolution of $0.316 \mathrm{~nm}$ covering a spectral range of $180-880 \mathrm{~nm}$. In the current experiments a spectral band of 350-800 nm was selected. A computer interface allows the user to vary the integration time from $5 \mathrm{~ms}$ to $10 \mathrm{~s}$ and analyze the recorded spectroscopic signal. The spectra were recorded during a time window of $100 \mathrm{~ms}$, so 500 pulses were integrated.

The samples were mainly ceramics such as silicon carbide $(\mathrm{SiC})$, aluminum nitride ( $\mathrm{AlN})$ and aluminum oxide $\left(\mathrm{Al}_{2} \mathrm{O}_{3}\right)$, although some other materials were tested as references, such as pure aluminum, copper and silicon.

The experiments were carried out at ambient pressure in all cases. The spectroscopic signals were analyzed with the aim of finding useful correlations between spectra and processing characteristics.

\section{Results and discussion}

Different gathering configurations were tested varying the acquisition angle, finding that acquiring the plasma signal on-axis produced the best repeatability and the most intense signal, presenting a better signal-to-noise ratio (SNR) as shown in Table 1.

Table 1 Signal-to-noise ratio for different acquisition angles for an AlN sample considering the average value of the signal in the broad band of $350-800 \mathrm{~nm}$

\begin{tabular}{lcccc}
\hline Acquisition angle & $90^{\circ}$ & $30^{\circ}$ & $20^{\circ}$ & $0^{\circ}$ \\
\hline Sinal-to-noise ratio & 15.0 & 5.2 & 4.6 & 2.8 \\
\hline
\end{tabular}

\subsection{Material identification feasibility}

The collected spectra of each material presented characteristic emission lines and intensities, identifiable features that can be distinguished from each other, which means that the system is capable of discriminating between different processed materials using different recognition algorithms, like linear or rank correlation.

As seen in Fig. 2 and table 2, the spectra from two samples having aluminum as major constituent show a similar shape in a broadband analysis. However, some differences can be observed if a narrowed spectral region is studied, as in the case of lines 3 and 7. These lines represent the enhanced emission of nitrogen and oxygen respectively, due to the sample interaction with air breakdown. At closer look, one will note the strong ion nitrogen plasma line (3) observed for AIN, while the O II plasma line (7) is registered during laser ablation of $\mathrm{Al}_{2} \mathrm{O}_{3}$ ceramic (and sapphire).

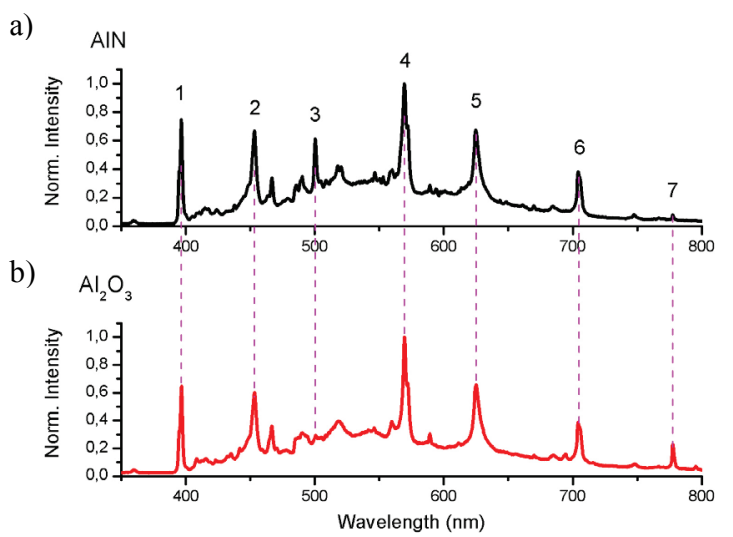

Fig. 2 Spectra of ceramics a) $\mathrm{AlN}$ and b) $\mathrm{Al}_{2} \mathrm{O}_{3}$ processed with ns laser pulses showing visible spectral differences.

Table 2. Emission lines showed in spectra of Fig. 2.

\begin{tabular}{ccc|ccc}
\hline Peak & Wavelength & Element & Peak & Wavelength & Element \\
\hline 1 & $396.15 \mathrm{~nm}$ & Al I & 5 & $624.33 \mathrm{~nm}$ & Al II \\
2 & $452.92 \mathrm{~nm}$ & Al III & 6 & $704.21 \mathrm{~nm}$ & Al II \\
3 & $500.27 \mathrm{~nm}$ & N II & 7 & $777.42 \mathrm{~nm}$ & O I \\
4 & $569.66 \mathrm{~nm}$ & Al III & & & \\
\hline
\end{tabular}

From Fig. 2 and Fig. 3 can be observed that the same emission lines are present in the pure aluminum sample (99.99\% of purity) and in $\mathrm{AlN}$ and $\mathrm{Al}_{2} \mathrm{O}_{3}$ ceramics although with different relative intensities. The same situation occurs with silicon and silicon carbide.

\subsection{Influence of the trepanning optics}


A comparison study was carried out to evaluate the effect of using a trepanning optics system during material processing and plasma signal acquisition.

The measurements demonstrated that more intense and better defined lines were detected using the trepanning optics system presenting better SNR than the signals acquired without, as shown in table 3 and in Fig. 3. By using the trepanning system, the focused laser beam can reach always a fresh surface, producing high intensity plasma.

Table 3. SNR of acquired signal for materials processed with and without mechanical laser trepanning system (considering the spectral region of $350-800 \mathrm{~nm}$ ).

\begin{tabular}{lcccc}
\hline & Pure $\mathrm{Al}$ & $\mathrm{AlN}$ & $\mathrm{Al}_{2} \mathrm{O}_{3}$ & $\mathrm{Si}$ \\
\hline Trepanning & 13.4 & 15.0 & 13.8 & 17.6 \\
No Trepanning & 1.9 & 3.3 & 2.3 & 3.4 \\
\hline
\end{tabular}

a)

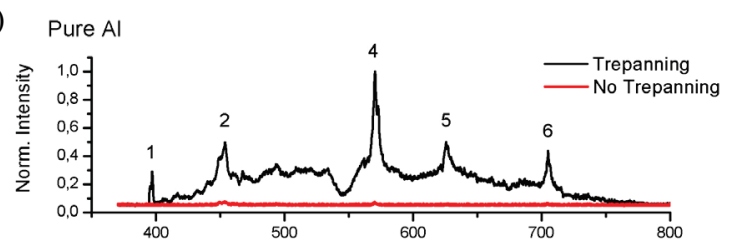

b)

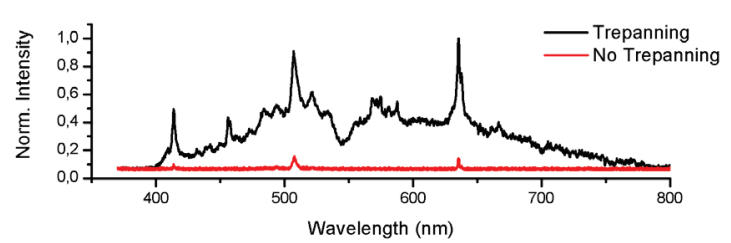

Fig. 3 a) Pure aluminum and b) silicon spectra gathered with and without the use of the trepanning system (same peaks as shown in Table 2).

Under the same processing conditions, it is clearly observed a better-defined spectrum when using the trepanning system.

\subsection{Influence of the focus position}

In order to find a strategy to automatically identify the correct focusing conditions by analyzing the induced plasma spectra, line processing was carried out while varying the lens-sample distance and acquiring plasma information. The $\mathrm{Z}$ position in the motion table was set to produce a focused spot above the surface, then the lens system was displaced downwards in controlled steps, until the laser focus was placed inside the bulk material. At each step plasma signal was recorded to identify its behavior. The trepanning system was used.

By modifying lens-sample distance, it was found that the intensity of the signal was at maximum when the focal point of the laser beam was placed at the surface or \pm 100 $\mu \mathrm{m}$ around it. The normalized intensity demonstrates a Gaussian profile while defocusing the beam in both directions, as seen in Fig. 4.

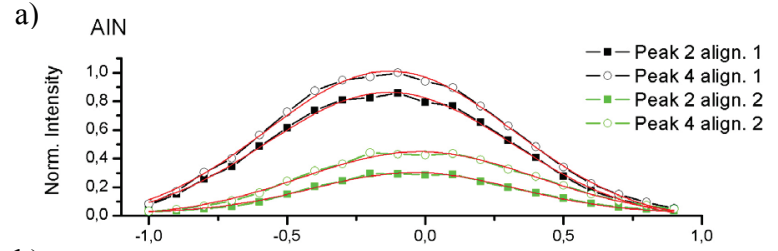

b) $\mathrm{Al}_{2} \mathrm{O}_{3}$ Distance from focus $(\mathrm{mm})$--Peak 2 align.

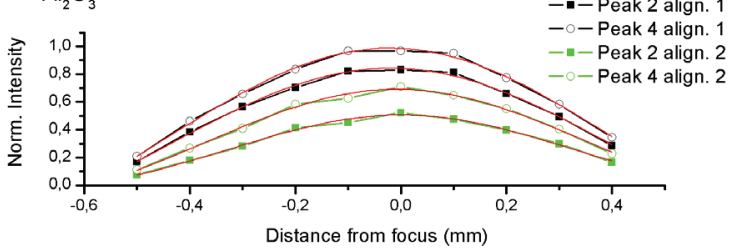

Fig. 4 Plasma intensity of selected lines 2 and 4 (as shown in Table 2), depending on focus position for two different alignments Ceramic a) $\mathrm{AlN}$ and b) $\mathrm{Al}_{2} \mathrm{O}_{3}$.

This result shows that by evaluating plasma emission signal while processing under the mentioned conditions, the best focusing characteristics can be determined by analyzing the signal intensity of selected emission lines and identifying its higher intensity. This strategy projects one straightforward option of applying a control based on the spectroscopic signal in a preselected spectral band.

\subsection{Linear correlation}

In order to analyze the degree of resemblance between different spectra and a reference signal, several groups have used the technique of correlation coefficient as a reliable identification algorithm when working with LIBS data $[2,6]$.

By applying

$$
r=\frac{\sum_{i=1}^{n}\left[x\left(\lambda_{i}\right)-\bar{x}\right]\left[y\left(\lambda_{i}\right)-\bar{y}\right]}{\sqrt{\sum_{i=1}^{n}\left[x\left(\lambda_{i}\right)-\bar{x}\right]^{2}} \sqrt{\sum_{i=1}^{n}\left[y\left(\lambda_{i}\right)-\bar{y}\right]^{2}}}
$$

a correlation coefficient $r$ is obtained which presents a maximum value of 1 when sample and reference are identical and a minimum of -1 when the signals have the same values but with opposite signs. $x\left(\lambda_{i}\right)$ and $y\left(\lambda_{i}\right)$ are the intensities of the emission signal for each wavelength $\lambda_{i}$ of the measured and reference spectra, respectively, while $\bar{x}$ and $\bar{y}$ are their mean values.

It is possible to compare a measurement with a set of pre-recorded data to identify a sample being processed with the aim of applying the best machining parameters for that specific material. During the variation of the focusing conditions, the correlation coefficient was calculated and the results are shown in Fig. 5.

For lens-sample distances close to on-surface focusing, the spectra of the same processed material present a high degree of similarity; meanwhile for different focusing conditions they become more disparate. Also when applying cross-correlation of samples with a very similar constitution, like $\mathrm{AlN}$ and $\mathrm{Al}_{2} \mathrm{O}_{3}$, with a very similar constitution in the $200-800 \mathrm{~nm}$ broad band, the algorithm does not present a good discriminating capability. It then delivers high coef- 
ficients, meaning great similarity. This situation can be overcome by identifying a reduced spectral region where the signals for different processed materials show a stronger contrast. In a preselected spectral region, the application of the identification method is more precise.

a)

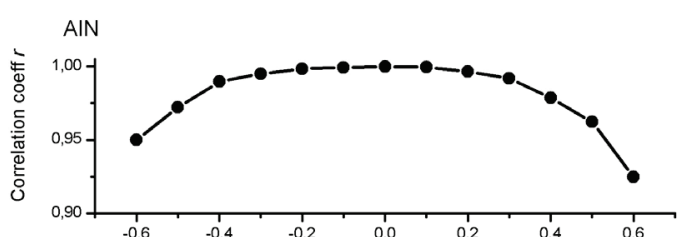

b)

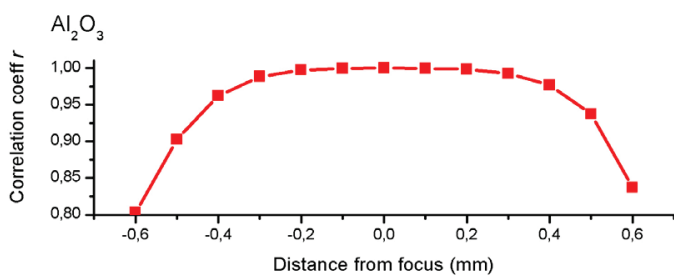

Fig. 5 Correlation coefficient while varying focusing position for a) $\mathrm{AlN}$ and b) $\mathrm{Al}_{2} \mathrm{O}_{3}$.

In the mentioned case the analyzed range is reduced to 495-505 $\mathrm{nm}$ or $772-782 \mathrm{~nm}$. The correlation coefficient technique now shows an improved performance, discriminating between two ceramics and other materials, as shown in table 4.

Table 4. Cross correlation among different materials.

\begin{tabular}{cccccc}
\hline \multicolumn{1}{c}{} & Pure $\mathrm{Al}$ & $\mathrm{AlN}$ & $\mathrm{Al}_{2} \mathrm{O}_{3}$ & $\mathrm{Si}$ & $\mathrm{SiC}$ \\
\cline { 2 - 6 } Pure $\mathrm{Al}$ & 1 & 0.31 & 0.73 & 0.16 & 0.22 \\
$\mathrm{AlN}$ & 0.31 & 1 & 0.25 & 0.63 & 0.58 \\
$\mathrm{Al}_{2} \mathrm{O}_{3}$ & 0.73 & 0.25 & 1 & 0.21 & 0.27 \\
$\mathrm{Si}$ & 0.16 & 0.63 & 0.21 & 1 & 0.89 \\
$\mathrm{SiC}$ & 0.22 & 0.58 & 0.27 & 0.89 & 1 \\
\hline
\end{tabular}

\subsection{Influence of crater depth}

The next step of the investigation was driving circles using the trepanning optics without changing $\mathrm{Z}$ position to drill a through-hole and read the plasma signal during the whole operation, looking forward to relating the intensity of the acquired signal and the processing depth.

a)

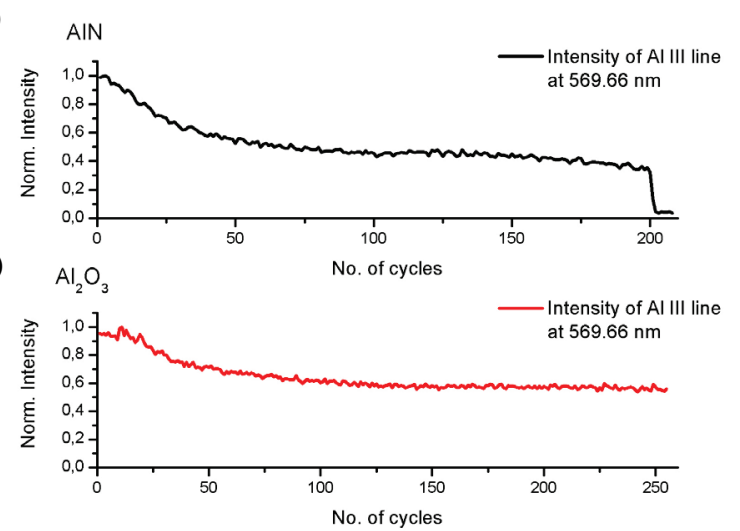

Fig. 6. Temporal evolution of a selected emission line while through-hole drilling. Each cycle represents a $100 \mathrm{~ms}$ period. Both samples, a) $\mathrm{AlN}$ and b) $\mathrm{Al}_{2} \mathrm{O}_{3}$, have a $300 \mu \mathrm{m}$ thickness. For b) the trough-hole was not completed.
Through-hole drilling was produced on ceramics and metals, and in both cases a decrease in plasma intensity took place as the number of laser pulses increased. After the initial intensity decrease, signal remained at a semiconstant level until the bottom of the hole was reached, where the intensity dropped to zero, as observed in Fig. 6.

According to the results shown in this section and in the previous one, by evaluating the plasma signal, the optimum focus position can be identified when the intensity of selected peaks reaches its maximum height. Also, in the case of drilling, when processing reaches the bottom of the sample a drop in the signal intensity is produced. Both features can be applied as control signal to automatically identify the starting and final processing points.

\subsection{Influence of delivered energy}

In order to find a relationship between power density and plasma intensity, line drawing was performed for different laser pulse energies.

When the delivered laser power was decreased so was the amount of removed material, and thus, the intensity of the plasma signal, as seen in Fig. 7.

a)

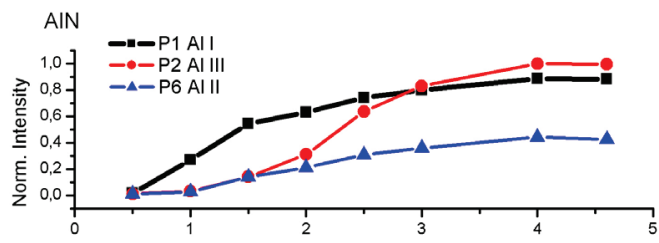

b)

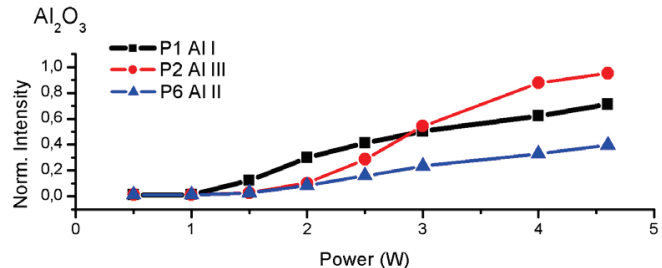

Fig. 7 Plasma intensity dependency on applied power (same peaks as shown in Table 2) for a) $\mathrm{AlN}$ and $\mathrm{Al}_{2} \mathrm{O}_{3}$ samples.

Non-ionized element lines demand less delivered energy to be emitted and as the energy increases ionized species become dominant, as observed in Fig. 7. This observation can occur due to an increase in thickness of the plasma, becoming opaque and creating self-absorption, plasmashielding at high density $[16,19]$. So, when low energy levels are to be used to produce machining on these materials, the main analytical interest should be focused on the non-ionized emission lines. Meanwhile for high energy levels, the ionized spectral lines become more important for a potential processing control strategy.

\section{Conclusions}

The results demonstrate the potential of LIBS as an online monitoring device to control laser material processing, due to the fact that all the parameters investigated in the present work influence the characteristics of emitted plasma, leading to basic relationships between spectroscopic signal and processing parameters.

Further research is being pursued to obtain a robust method utilizing the existent correlation between laser machining and plasma analysis to further improve the quality 
and efficiency in precision micro fabrication.

The next stage of the project is related to the use of a picosencond pulsed laser as an excitation tool in order to establish a comparison with previous results. In addition, the effects oat different laser wavelength, second and third haronic of the fundamental wavelength, will be investigated in detail.

\section{Acknowledgments}

The authors want to thank the funding of the German Academic Exchange Service (DAAD). Valuable help was provided by Corinna Nock during the data analysis. Part of this study is supported by the AiF program "Förderung von Vorlaufforschung bei Wachstumsträgern in benachteiligten Regionen" project number VF081026.

\section{References}

[1] Cramers, D. „Handbook of Laser-Induced Breakdown Spectroscopy“. John Wiley. 2006.

[2] J.M. Vadillo and J.J. Laserna: Spectrochim. Acta B 59, (2004) 147-161.

[3] T.A. Labutin, A.M. Popov, V.N. Lednev and N.B. Zorov: Spectrochim. Acta B 64, (2009) 938-949.

[4] M. Lentjes, K. Dickmann and J. Meijer: J. Appl. Phys. A 88, (2007) 661-666 (5).

[5] I.B. Gornushkin, B.W. Smith, H. Nasajpour and J.D. Winefordner: Anal. Chem. 71 (1999), 5157-5164.

[6] E. Tzamali and D. Anglos: "Lasers in the Conservation of Artworks" ed. by J. Nimmrichter, W. Kautek and M. Schreiner, (Springer, Heidelberg, 2007) p. 377.

[7] T. Tong, J. Li and J.P. Longtin: App. Opt. 43, (2004) 1971.

[8] M. Lentjes, K. Dickmann and J. Meijer: Spectrochim.
Acta B 62, (2007) 56-62.

[9] T.M. Moskal and D.W. Hahn: Appl. Spectrosc. 56 (2002) 1337-1344

[10] M. Gaft, I. Sapir-Sofer, H. Modiano and R. Stana: Spectrochim. Acta B 62, (2007) 1496-1503.

[11] M.D. Mowery, R. Sing, J. Kirsch, A. Razaghi, S. Bechard and R.A. Reed: J. Pharma. \& Biomed. Anal. 28, (2002) 935-943.

[12]F.J. Fortes, L.M. Cabalin and J.J. Laserna : Spectrochim. Acta B 63, (2008) 1191-1197.

[13] H. Balzer, M. Hoehne, V. Sturm and R. Noll: Spectrochim. Acta B 60, (2005) 1172-1178.

[14] H. Balzer, M. Hoehne, R. Noll and V. Sturm: Anal. Bioanal. Chem. 385 (2006), 225-233.

[15]T.V. Kononenko, D. Walter, V.I. Konov and F. Dausinger: Quantum Electron 39, (2009) 328-332.

[16] J.A. Aguilera, C. Argon and F. Penalba: App. Surf. Sci. 127-129, (1998) 309-314.

[17] R. Noll, I. Monch, O. Klein and A. Lamott: Spectrochim. Acta B 60, (2005) 1070-1075.

[18] V. Bulatov, L. Xu and I. Schechter: Anal. Chem. 68, (1996) 2966-2973.

[19] M. Ying, Y. Xia, Y. Sun, M. Zhao, Y. Ma, X. Liu, Y. Li and X. Hou: Laser \& P. beams 21, (2003) 97-101.

[20] J. Li, J. Lu, Z. Lin, S. Gong, C. Xie, L. Chang, L. Yang and P. Li: Opt. \& Laser Tech. 41, (2009) 907913.

[21] M. Schwagmeier, N. Mueller and D. Ashkenasi: Proceedings of the Fifth International WLT-Conference on Lasers in Manufacturing 2009.

(Received: June 07, 2010, Accepted: July 06, 2011) 